\title{
VARIACIONES CULTURALES EN EL VALLE DE LIMA DURANTE LA OCUPACIÓN INCAICA
}

\author{
CULTURAL VARIATIONS IN LIMA VALLEY \\ DURING THE INKA PERIOD
}

\author{
Luisa Díaz* y Francisco Vallejo **
}

\begin{abstract}
El presente trabajo busca dar cuenta de los profundos cambios culturales en el área de Lima, durante la conquista Inka. Partimos del estudio de uno de los centros urbanos más importantes de ese período, conocido actualmente como Armatambo y del cementerio arqueológico denominado La Rinconada. Se precisan los elementos culturales característicos del señorío Ichma, unidad política que administraba la región de Lima antes de la llegada de los Incas y que gobernó desde el centro religioso Pachacamac. En este contexto, segregaremos el material cultural de las poblaciones locales, en los sitios antes mencionados, para compararlos con los patrones culturales incaicos, y a partir de lo cual se establecen los rasgos básicos Ichma e Inka, expresados en la arquitectura, la presencia de elementos extra regionales, la cerámica y el patrón funerario.
\end{abstract}

Palabras claves: señorío Ichma, Inka Regional, Lima, Pachacamac, Armatambo, La Rinconada.

This study explores the profound cultural changes that occurred in the Lima area during the Inca conquest. We investigated one of the most important urban centers currently known as Armatambo, and the cemetery La Rinconada. We describe the cultural elements that characterize the señorio Ichma, the political unit that had governed the Lima region from the religious center of Pachacamac since before the Inca conquest. We differentiate the cultural material of the local Ichma group, and compare it with Inka cultural patterns. We focus upon the following attributions: architecture, extra-regional elements, ceramics, and funeral patterns.

Key words: señorio Ichma, Regional Inka, Lima, Pachacamac, Armatambo, La Rinconada.

Aunque el período Inka es uno de los más conocidos en términos arqueológicos e históricos, es claro que aún existen fuertes discrepancias en torno al momento exacto en el cual empezó su expansión en los Andes y, en especial, hacia la costa central peruana. La mayoría de los investigadores han asumido la fecha de ocupación de la región de Lima hacia la segunda mitad del siglo XV (Bueno 1982; Eeckhout 1995; Negro y Fuente 1989; Silva 1992). Si bien hay concordancia en que la expansión del imperio incaico afecta la costa central peruana durante el reinado de $\mathrm{Pa}$ chacutec, la época de dicha expansión abarca un espacio de tiempo variable, entre finales del siglo XIV y comienzos de la segunda mitad del siglo XV. Este hecho puede ser de vital importancia, dado que al presente las investigaciones arqueológicas indicarían una fuerte presencia cultural de los patrones estilísticos incaicos en la costa central peruana, lo que no se puede explicar por una presencia Inka de tiempo tan reducido, menor a 100 años. Cabe pensar entonces en la posibilidad de que la presencia Inka haya sido más larga en el tiempo a lo comúnmente aceptado, o que se haya dado con mayor intensidad a la estimada, de tal manera que permitió que los patrones locales se modificaran.

Las investigaciones efectuadas por nosotros en el valle de Lima en los sitios arqueológicos de Armatambo y La Rinconada evidencian una ocupación del Intermedio tardío (1.000 a 1.460 d.C., correspondiente al señorío Ichma) y del Horizonte Tardío (1.460 a 1.533 d.C., correspondiente a la ocupación Inka). Esta secuencia demuestra cambios en los rasgos culturales locales frente a los nuevos patrones Inka. Además, los patrones culturales y estilísticos que componen esta secuencia, en especial algunos estilos cerámicos, hasta ahora habían sido reportados como preincaicos, descartando el influjo Inka.

\section{El Señorío Ichma y la Ocupación Incaica}

Según las fuentes etnohistóricas, antes de la conquista Incaica de la costa central peruana floreció el señorío Ichma en los valles del Rimac y Lurín (Rost-

\footnotetext{
* Universidad Nacional Mayor de San Marcos; Av. Caminos del Inca 2192, Surco, Lima, Perú. ledarriola@ yahoo.es

** Proyecto Arqueológico Armatambo, Jr. Camaná 233-A, Chorrillos, Lima, Perú. f_vallejob@yahoo.com
} 
worowski 1977). Su nombre al parecer también designó su centro político-religioso, del cual el asentamiento de Armatambo fue uno de sus centros más importantes (Albornoz 1967; Cobo 1882 [1639]). Sin embargo, se sabe que, a la llegada de los Inkas al valle de Lurín, la capital Ichma fue cambiada de nombre por el de Pachacamac (Rostworowski 1977:199). Esta unidad política, que floreció desde inicios del siglo XII hasta mediados del siglo XV, se regía bajo el dominio del centro religioso de Pachacamac, que durante la ocupación Incaica cobró aún mayor importancia, llegando a incorporar sus dioses regionales dentro del panteón de dioses Inka. Esta gran relevancia religiosa se mantuvo hasta la llegada de los españoles a mediados del siglo XVI.

Dentro del ámbito territorial mencionado se desarrollaron otros centros urbanos Ichma de menor jerarquía, como Armatambo (Hyslop y Mujica 1992), Puruchuca, Puruchuco, Rinconada Alta, por mencionar algunos, todos articulados entre sí por un eficiente sistema vial. La presencia Inka en todos estos lugares significó grandes cambios en el uso de los espacios, en la edificación de nuevas y grandes construcciones, en el cambio de los patrones funerarios, en el mayor acceso a productos extrarregionales, en especial de la región norteña, y en la presencia de nuevos tipos cerámicos, que en conjunto evidencian el nuevo orden político al que estuvieron sometidos. En este esquema, las investigaciones arqueológicas han venido dando cuenta de múltiples indicadores que constatan la presencia Inka, sin que se haya puesto mayor atención en definir arqueológicamente las características culturales del período precedente.

\section{Elementos Diagnósticos del Señorío Ichma}

Los trabajos arqueológicos realizados en Armatambo (Díaz y Vallejo 2002, 2003, 2004; Vallejo 1988) y La Rinconada (Díaz 2002), en la zona de Lima durante la década de 1990, y en particular en los últimos años, han coincidido en presentar el mismo problema de identificación de los contextos atribuibles a la cultura Ichma. Es claro que la investigación arqueológica poco ha contribuido en la definición de esta unidad cultural, sobre todo en términos de su tipología cerámica.

\section{Arquitectura}

Los materiales y las técnicas arquitectónicas de los períodos Ichma e Inka en Armatambo pre- sentan distinciones arquitectónicas que son bastante claras e incluso superpuestas en los registros de excavación. En Armatambo se evidencia la superposición de las técnicas arquitectónicas, específicamente el adobe rectangular sobre el tapial. Considerando la asociación de estas técnicas con otros materiales culturales, ha sido posible definir claramente la relación de cada una de estas técnicas con el período Inka o Ichma. La arquitectura Ichma utiliza recurrentemente el tapial, tanto en la arquitectura masiva como en la construcción de recintos y pasajes interurbanos. En nuestras recientes excavaciones en el área central de Armatambo la arquitectura del tapial está siempre subyacente a la arquitectura del adobe rectangular o paralelepípedo y asociada con cerámica Ichma. Los recintos Ichma son de planta ortogonal, con muros de tapial que en promedio debieron superar los dos metros de altura. Existen dos tipos principales de muros, los muros con un ancho promedio de 60 $\mathrm{cm}$ empleados en recintos internos de tamaño pequeño ( 3 × 3 m en promedio) y los muros de 90 a $100 \mathrm{~cm}$ que son utilizados como muros principales o ejes de las estructuras a los cuales se agregan los muros de $60 \mathrm{~cm}$. Los muros anchos también se utilizan para definir grandes espacios abiertos y lugares de tránsito.

La posición de los tapiales ${ }^{1}$ casi siempre es vertical y con escasa cimentación, en cambio, cuando los tapiales se adosan como reforzamientos sucesivos suelen tener una posición oblicua. Es importante advertir que algunos muros de tapial emplean un tipo particular de adobes rectangulares en su interior, los que suelen ser achatados y con una altura promedio de 8 a $10 \mathrm{~cm}$, con lados parcialmente descuadrados y con la superficie alisada, salvo en uno de sus lados, indicando que éstos fueron elaborados directamente sobre el suelo. Su utilización dentro de los muros de tapial es horizontal, formando algunas hileras independientes en el muro, en algunos casos llegando hasta tres, quizás a manera de amarre suplementario. La posición de los adobes dentro del tapial suele ser en doble fila horizontal, a veces dispuestos en la base misma y en otros casos en la parte media del muro. Estos muros en buen estado de conservación evidencian un fino enlucido de barro como acabado final. En algunos casos también se han encontrado evidencias de pisos enlucidos en los recintos más elaborados. Generalmente, los cambios de altura entre los diversos 
niveles arquitectónicos eran resueltos con rampas de ángulo variable o "planos inclinados" como señala Iriarte (1960). En La Rinconada, pese al estado de conservación del sitio, también hemos hallado evidencias del empleo del tapial como técnica constructiva, de este extenso asentamiento del mismo período.

Para la época Inka, las técnicas constructivas cambian radicalmente, pues se emplean frecuentemente los adobes paralelepípedos o adobes rectangulares del tipo Inka (Tello 1999:34). Estos adobes son de varios tamaños, aunque la altura promedio fluctúa entre los 12 y $14 \mathrm{~cm}$. Las improntas en estos adobes evidencian el uso de gavera, quedando siempre el lado superior del adobe con huellas de manos producidas al emparejar la mezcla de barro dentro de la gavera. Los adobes Inka presentan algunas inclusiones en la mezcla arcillosa, como pequeñas piedras, fragmentos de moluscos o pedazos de cerámica en pequeñas proporciones.

Entre los edificios donde recurrentemente se emplea este tipo de adobes se encuentran las pirámides con rampa de Pachacamac (Eeckhout 1995, 1999; Franco 1993; 1998; Paredes y Franco 1987) y una que recientemente hemos excavado en Armatambo (Díaz y Vallejo 2003) (Figura 1). De acuerdo con estas evidencias, varios de

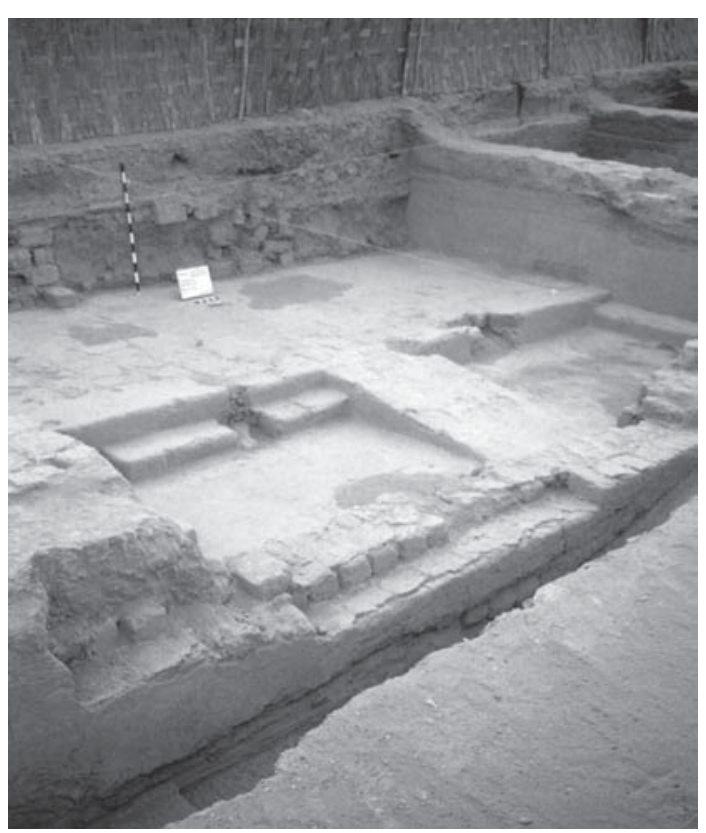

Figura 1. Patio en U de la Pirámide con rampa (SP1-AR), Armatambo.

$U$-shape patio of the pyramid with ramp (SP1-AR) at Armatambo. estos edificios serían cronológicamente pertenecientes al período Inka, lo que ciertamente es una afirmación discutible, ya que hasta el momento diversos investigadores les han asignado una cronología exclusiva del Intermedio tardío. Creemos, en todo caso, que algunas interpretaciones en torno a la presencia Inka en las pirámides con rampa deben ser revisadas y no considerar la ocupación Inka sólo secundaria o intrusiva a este tipo de edificios.

\section{El mullu}

El mullu (Spondylus princeps) puede ser un gran indicador cronológico para establecer la aparición del Estado incaico en la región Ichma y el desarrollo del mismo. De acuerdo con nuestras investigaciones, es claro que en los contextos Ichma e Inka la presencia de Spondylus no es homogénea. En los contextos del período Inka el mullu aparece con una notable presencia. En los contextos funerarios Ichma suele ser mínima o ausente en numerosos grupos de tumbas. Aún los productos derivados de Spondylus, como las cuentas de chaquira, son escasos. Este hecho podría interpretarse de varias maneras, teniendo en cuenta el culto que se ejercía a Pachacamac y a una posible centralización de los elementos rituales y de ofrenda en manos de la casta sacerdotal. De otro lado, también podría interpretarse como indicios de una fuerte elitización en la sociedad Ichma, donde el uso del mullu no alcanzó a los estratos inferiores, pero contrariamente a esto se ha apreciado que en los contextos funerarios Ichma con mayor nivel de elementos asociados (incluso con más de 30 vasijas de cerámica) tampoco suele aparecer este bivalvo.

Creemos más bien que esto obedece a varias razones; por un lado, la ausencia del mullu en los contextos Ichma preincaicos indica una fuerte limitación a la disponibilidad de este elemento para las diferentes prácticas sociales locales. Es mucho más probable que los niveles de intercambio con la costa norte hayan sido escasos y que por consiguiente el volumen de mullu obtenido represente una cantidad muy modesta, si lo comparamos con los contextos del período Inka, donde se presenta en grandes cantidades. En apoyo a esta hipótesis, podemos afirmar que el mullu no es el único elemento que registra una escasa presencia en los contextos Ichma, sucede lo mismo con los metales, 
limitándose a pequeños objetos suntuarios, y con los pigmentos y otros productos exóticos, como los moluscos de las familias Coneidae (Conus sp.) y Ostreidae (Ostrea sp.). Esto reforzaría nuestra hipótesis de que los Ichma tuvieron bajos niveles de intercambio con sociedades extrarregionales. En este contexto, la idea de un culto muy extendido de Pachacamac antes de su incorporación al panteón incaico debe ser revisada y analizada en base a los datos arqueológicos disponibles. Todo parecería indicar más bien que luego de la sujeción de los pueblos de la región norte del Perú y del Ecuador, la administración incaica desplegó una eficaz red de transporte del mullu y otros productos norteños, poniendo en ejecución todos los medios disponibles para mantener un tránsito fluido hacia todo el Imperio. En el caso de Armatambo, los datos arqueológicos indicarían que este sitio fue usado intensamente en la ruta del mullu y otros productos exóticos en tránsito hacia Pachacamac, teniendo en cuenta que es el puerto más cercano al famoso santuario y es atravesado por el "Camino de los Llanos" (Cobo 1956 [1653], 1882 [1639], en Fernández de Oviedo 1945 [1547]). Es posible que la ubicación privilegiada de Armatambo hiciera que adquiriera mayor estatus económico y social durante la época Inka, constituyéndose en un foco de crecimiento para un nuevo grupo de mercaderes locales de elevada posición económica.

\section{Los contextos funerarios}

La información obtenida en Armatambo y La Rinconada, luego de haber excavado alrededor de 306 contextos funerarios, nos ha permitido definir las características generales del patrón funerario Ichma durante el Intermedio tardío y el Horizonte tardío. Esta información se complementa con las reportadas para sitios como Pachacamac (Uhle 1903), Puruchuco, Huaquerones, El Sauce, Santa Cruz y otros. Tal parece, que las características generales del patrón funerario presentan pocos cambios durante las fases Temprana y $\mathrm{Media}^{2}$ de la secuencia cultural Ichma, pues las variantes son relativamente pocas y pareciera más bien que los cambios son esencialmente graduales. Entre los varios elementos comunes definidos en el patrón funerario Ichma podemos mencionar que las tumbas son elaboradas mediante una cista de planta circular y contorno simple, dentro de la cual se acondicionan a los individuos enfardelados (Figura 2). El fardo generalmente presenta relleno de te-

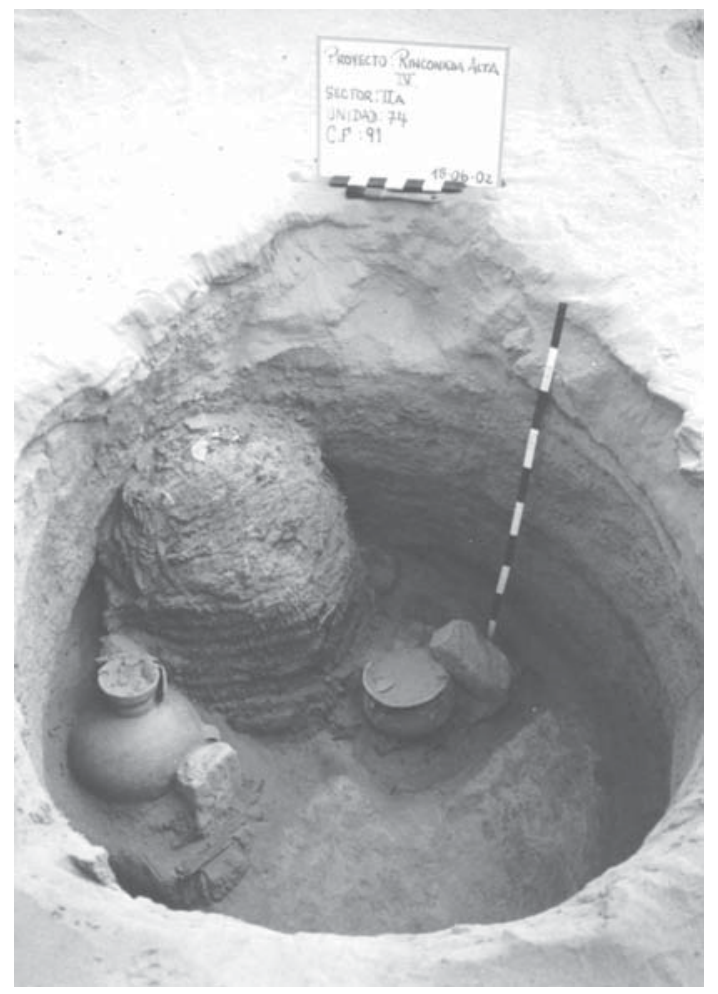

Figura 2. Contexto funerario Ichma, La Rinconada. Ichma funerary context, La Rinconada.

las o vegetales, donde pueden disponerse objetos suntuarios como pequeños metales o elementos de semillas a la altura del rostro, pecho y muñecas.

En Armatambo la orientación básica de los individuos es hacia el suroeste, hacia Pachacamac. $\mathrm{Al}$ respecto, es importante señalar que en el caso de La Rinconada los individuos se orientan hacia el este, probablemente siguiendo un antiguo camino que llevaba al oráculo. Los cuerpos, por lo general, suelen mantener una posición completamente flexionada tanto en niños como adultos; mientras que los neonatos siempre están en posición extendida. Los elementos externos asociados al fardo siempre se encuentran ubicados en semicírculo delante del individuo, que en algunos casos pueden ser de hasta dos niveles superpuestos. Es recurrente el uso de mates ${ }^{3}$, los pequeños se utilizan como coberturas de las bocas de las vasijas, y casi siempre se observa el empleo de un mate de gran diámetro con ceniza en su interior, que es puesto como base $\mathrm{o}$ asiento de los individuos adultos. También se observa el uso de pigmento rojo en el rostro de los individuos adultos, o en el área correspondiente del fardo. 
Respecto de la disposición de las extremidades de los individuos adultos, es también recurrente que los brazos estén pegados al pecho, mientras que la disposición del pie derecho sea sobre el izquierdo. Es también recurrente la presencia de grupos de pequeños carrizos dispuestos al interior del fardo, ubicados por lo general a cada lado del individuo en grupos de dos o de cuatro, tanto en su lado derecho como el izquierdo. A todo esto se agrega la preferencia de ubicar las zonas de cementerio alejadas de las áreas con presencia de arquitectura, constituyéndose cementerios alejados de las áreas de uso permanente.

Durante el período Inka, las variantes en el patrón funerario suelen ser más numerosas, abundando las tumbas colectivas y prefiriéndose la elaboración de algunas estructuras que recubren o se adosan a las paredes de la cista o, en su defecto, reacondicionando antiguos espacios de arquitectura Ichma e incluso Inka.

Las estructuras funerarias del período Inka son en la mayoría de los casos de planta rectangular y reutilizan, por lo general, adobes para delimitar el contorno de la cista, dispuestos sin argamasa, lo que les da una configuración rústica y endeble. Muchas de las tumbas de este período son de carácter intrusivo a los contextos Ichma originales. Las tumbas del período Inka se ubican de forma aleatoria, en espacios con arquitectura Ichma y rellenados en esa época. Cuando las tumbas son individuales, por lo general, la cista es de planta circular con un promedio de $90 \mathrm{~cm}$ de diámetro, utilizándose espacios con arquitectura (Vallejo 1988). En cierta manera, los cementerios Inka buscaron la cercanía a áreas arquitectónicas evitando, en lo posible, la utilización de áreas estériles. Resalta en este período el acompañamiento de un ajuar funerario más elaborado y diverso, con ofrendas que denotan posición social y/o actividades productivas (Díaz y Vallejo 2004). En las tumbas de mayor jerarquía la presencia de elementos extrarregionales es una constante, evidenciado por la presencia de moluscos tropicales, metales preciosos, plumas de aves y maderas provenientes de la selva, entre otros.

\section{La cerámica}

Los grupos estilísticos cerámicos, que confluyen durante el período Inka en el valle de Lima, se encuentran limitados a cuatro grupos principales: el estilo Inka cuzqueño, el estilo Ichma, el estilo Inka regional y el estilo Chimú-Inka. Cada uno de ellos con características propias, pero en muchos casos, salvo el estilo Inka cuzqueño, compartiendo elementos debido al fuerte contacto e interacción que se produce entre ellos durante este período.

Definitivamente, el grupo más numeroso y de mayor presencia lo constituye el estilo Ichma en su fase Tardía, seguido por el estilo Inka regional. Este hecho es una clara evidencia de que los talleres cerámicos Ichma y las prácticas productivas locales se mantuvieron en gran medida adaptándose a la nueva situación social; aunque también se destacan varios elementos novedosos, en especial la incorporación de nuevas y mejores técnicas artesanales.

El estilo Ichma, en proceso de definición (Bazan 1990; Bonavía 1959; Díaz y Vallejo 2002; Eeckhout 1995, 1998, 1999; Feltham 1983; Franco 1998; Iriarte 1960; Paredes y Ramos 1994; Silva $1992)^{4}$, presentaría varias fases, en cuyas fases intermedias se conformarían sus características más relevantes. Estas piezas mantuvieron cierta uniformidad técnica, como cuerpos globulares, decoración pintada restringida a dos o máximo tres colores, siempre con un acabado mate. Los acabados en ambiente reductor suelen ser escasos, mientras que el ambiente de cocción oxidante completo fue más común. Incluso, en cuanto a la morfología, los platos y los cuencos son prácticamente ausentes en el conjunto cerámico Ichma. Las formas decorativas o decoraciones modeladas más típicas están limitadas a vasijas con representación de cucurbitáceas, aves y un tipo muy particular de representación antropomorfa conocido como "caragollete" (Bonavía 1959) (Figura 3). Los motivos se muestran igualmente repetitivos utilizando líneas zigzagueantes y estilizaciones de peces. Existen, por supuesto, varios otros elementos que escapan a este patrón, pero su presencia es muy escasa.

El estilo Inka regional, en cambio, demuestra un mejor y más variado tratamiento en los tipos cerámicos. Muchos de ellos provienen del estilo Ichma, pero adecuados a las nuevas técnicas productivas y decorativas disponibles, conformando un estilo anexo. De esta forma, el Inka regional está compuesto de atributos estilísticos y morfológicos compartidos del estilo Ichma y varios de los estilos que los inkas absorben o toman de la región central del Perú y en algunos caso de la región Chimú. Sin embargo, se hace evidente una centralización productiva existiendo varios tipos cerámicos muy estandarizados, tanto en forma, dimen- 


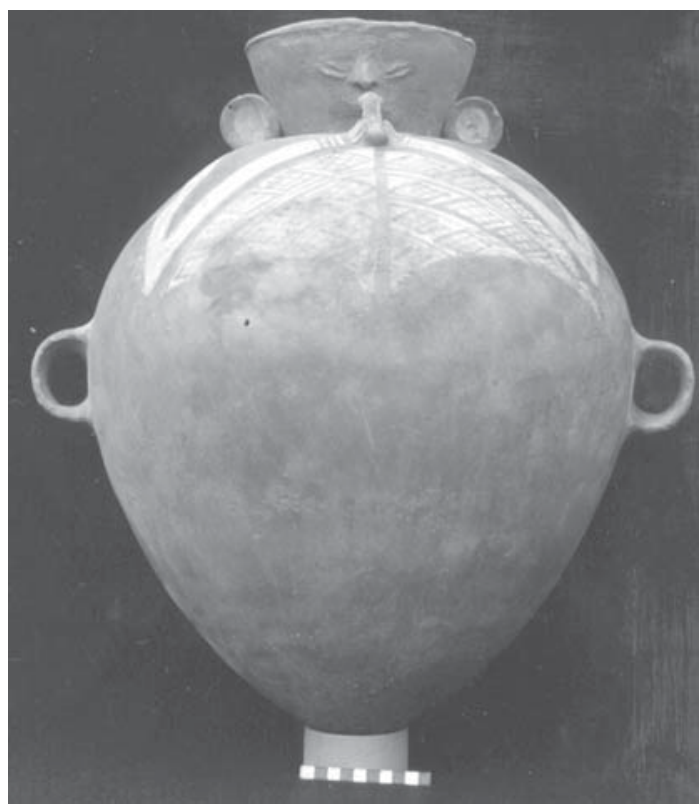

Figura 3. Vasija cara-gollete del estilo Ichma. Armatambo Neck-face vessel, Ichma style, from Armatambo.

sión como acabados. Es evidente que para la producción de los tipos cerámicos pertenecientes a este estilo debieron existir talleres cerámicos especializados afincados en la propia región Ichma, desde donde se distribuían los especímenes. Un elemento que destaca en la posible función de estos especímenes cerámicos, es la presencia de estas piezas en contextos culturales donde se evidencia una mayor jerarquía social.

\section{Conclusiones}

Las variaciones culturales que se producen en el valle de Lima en el tránsito de la sociedad local Ichma a la sociedad Inka están en la mayoría de los casos circunscritas a los propios cambios que se producen al interior de un mismo grupo humano. De hecho, la suplantación de elementos culturales está limitada a muy pocos aspectos y más bien se evidencia un lento pero progresivo cambio de costumbres, así como un reacomodo o readaptación de las costumbres locales en vista de los nuevos elementos Inka. Si bien debió existir algún porcentaje poblacional de origen foráneo, como los mitimaes citados por diversos cronistas, y quizás también un grupo de estricto origen cuzqueño, éstos debieron ser numéricamente menores en comparación a la población Ich- ma, que dentro de este nuevo orden político y social mantuvo su propia dinámica cultural incorporando estos cambios.

La percepción que se tiene del período Inka en el valle de Lima, a partir de los datos arqueológicos, es por tanto una incorporación vertical paulatina de nuevos elementos culturales y no de una suplantación de los mismos. De esta manera son explicables tanto la continuidad de una tradición Ichma como el surgimiento de formas mixtas entre lo Ichma y lo Inka (Figura 4).

Los Inkas, en su estrategia de dominación fueron tan genéricos como diversos. Genéricos porque aplicaron un mismo sistema organizativo de gobierno y un esquema tributario uniforme para cada región o provincia anexada; diversos porque a su vez tuvieron un tratamiento muy particular de imposición cultural y social en cada provincia. En la región o provincia Ichma (Cornejo 2000), estos aspectos se muestran claramente, pues a la par del

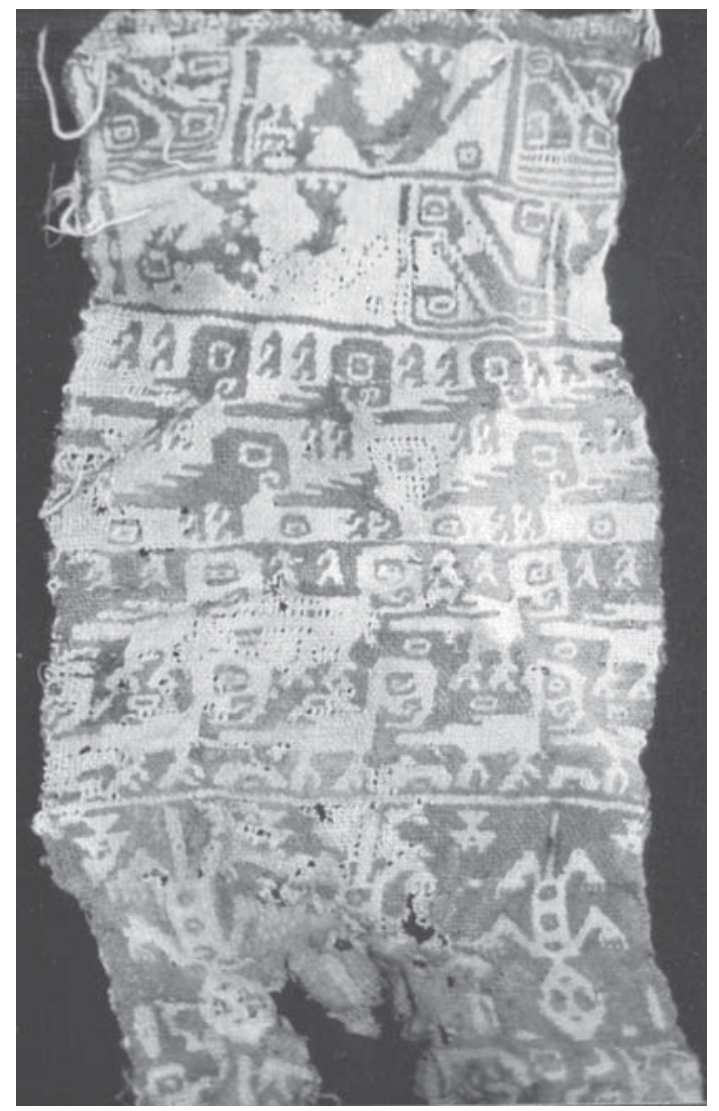

Figura 4. Textil con representaciones iconográficas Ichma e Inka. Armatambo

Textile with Ichma and Inka iconography from Armatambo. 
estricto sistema organizativo implantado para la región, en el caso de los sistemas de control y administración del imperio, fueron también bastante permeables con los elementos culturales locales. Finalmente, un aspecto que destaca en los contextos del período Inka es el de una mayor jerarquiza- ción y diferenciación especializada de la producción en la sociedad Ichma. En las tumbas, este elemento es particularmente notorio, pues se suele acompañar los artefactos que denotan la actividad de los individuos, como los signos de su posición y jerarquía social.

\section{Referencias Citadas}

Albornoz, C. de

1967 La Instrucción para Descubrir las Guacas del Pirú y sus Camayos y Haziendas. Journal de la Societé des Americanistes. Tomo LVI.

Bazan del Campo, F.

1990 Arqueología y etnohistoria de los períodos prehispánicos tardíos de la costa central del Perú. Tesis de Licenciatura, Escuela de Arqueología de la Universidad Nacional Mayor de San Marcos.

Bonavía, D.

1959 Cerámica de Puerto Viejo (Chilca). Actas del II Congreso Nacional de Historia del Perú, 137-168. Lima.

Bueno, A.

1982 El Antiguo Valle de Pachacamac. Espacio, Tiempo y Cultura. Boletín de Lima 4 (24).

Cobo, Fr. B.

1956 [1653] Historia del Nuevo Mundo. Editado por Biblioteca de Autores Españoles. Madrid.

1882 [1639] Historia de la Fundación de Lima. Impresión Liberal. Lima.

Cornejo, M.

2000 La nación Ischma y la provincia Inka de Pachacamac. Arqueológicas 24:148-173.

Díaz, L.

2002 Proyecto de Rescate Arqueológico: Rinconada Alta IV Etapa. Manuscrito presentado al Instituto Nacional de Cultura. Lima.

Díaz, L. y F. Vallejo

2002 Identificación de Contextos Ichma en Armatambo. Arqueología y Sociedad 14:47-75.

2003 Hallazgo de pirámides con rampa en Armatambo. Medio de Construcción 175:49-54.

2004 Armatambo y el dominio incaico en el valle de Lima. Boletín de Arqueología PUCP 6 (Primera parte): 355-374.

Eeckhout, P.

1995 Pirámide con rampa No 3 de Pachacamac, costa central del Perú. Resultados preliminares de la primera temporada de excavaciones (zonas 1 y 2). Boletín del Instituto Francés de Estudios Andinos 24: 65-106.

1998 Offrandes funéraires a Pachacamac et Pampa de las Flores. Exemples des relations entre les cotes nord et centrale du Perou a 1 époque pré-Inka. Baessler-Archiv Neue Folge 46: 165-229.

1999 Pirámide con rampa No III, Pachacamac. Nuevos datos, nuevas perspectivas. Boletín del Instituto Francés de Estudios Andinos 28:169-214.

Feltham, J.

1983 The Lurín Valley, Perú, A.D. 1000-1532. Ph.D. Thesis. University of London.
Fernández de Oviedo y G. Valdés 1945 [1547] Historia General y Natural de las Indias. Tomo XII. Editorial Guaranía, Asunción.

Franco, R.

1993 Los dos templos principales de Pachacamac. Revista del Museo de Arqueología 4:55-77.

1998 La Pirámide con Rampa $N^{o} 2$ de Pachacamac. Excavaciones y Nuevas Interpretaciones. Trujillo, Perú.

Hyslop, J. y E. Mujica

1992 Investigaciones de A.F. Bandelier en Armatambo (Surco). Gaceta Arqueológica Andina 22:63-86.

Iriarte, $\mathrm{F}$.

1960 Algunas apreciaciones sobre los Huanchos. Antiguo Perú; Espacio y Tiempo: 259-263. Editorial Juan Mejía Baca. Lima.

Negro, S. y M. del C. Fuentes

1989 Nieve-nieve: arquitectura y urbanismo en la costa central del Perú. Boletín de Lima 62:57-71.

Paredes, P. y R. Franco

1987 Pachacamac: las pirámides con rampa. Cronología y función. Gaceta Arqueológica Andina 13: 5-7.

Paredes, P. y J. Ramos

1994 Excavaciones arqueológicas en el sector Las Palmas, Pachacamac. Boletín de Lima 16:91-96.

Rostworowski, M.

1977 Breve Ensayo sobre el Señorío de Ychma. Etnía y Sociedad. Costa Peruana Prehispánica: 197-210. Instituto de Estudios Peruanos, Lima.

1978 Los Señoríos Indígenas de Lima y Canta. Instituto de Estudios Peruanos, Lima.

Rowe, J.H.

1962 Stages and periods in archaeological interpretation. Southwestern Journal of Anthropology 18:40-54.

Silva, J.

1992 Ocupaciones postformativas en el valle del Rimac: Huachipa-Jicamarca. Pachacamac 1:49-74.

Tello, J.C.

1999 Arqueología del Valle de Lima. Cuadernos de Investigación del Archivo Tello 1:23-138. Museo de Arqueología y Antropología. Universidad Nacional Mayor de San Marcos.

Uhle, $\mathrm{M}$.

1903 Pachacamac. Peruvian Expedition of 1896. Department of Archaeology, University of Pennsylvania, Philadelphia.

Vallejo, F.

1988 Ritos funerarios en el Perú prehispánico. Análisis de una tumba del complejo funerario de Armatambo, Lima. Ande: Prehistoria Come Progetto. Documento de EtnoArchaeología Precolombina. Università Católica dil Sacro Cuore. 


\section{Notas}

1 Sinónimo de tapia. Se trata de un muro de tierra encofrada y apisonada. También se refiere a un conjunto de paredes de tapia.

2 Tomando como base el material cerámico, hemos clasificado la secuencia cultural Ichma en tres fases principales, Ichma temprano, medio y tardío. (Díaz y Vallejo 2002).

3 El mate es un tipo de calabaza (Lagenaria sp.) que por lo general se usa para contener líquidos o sólidos. En los contextos Ichma los mates reemplazan a los cuencos y platos.
La mayoría son de textura lisa sin decoración, pero algunos presentan diseños pirograbados.

4 La cerámica de estilo Ichma era conocida indistintamente como estilo Huancho, estilo Puerto Viejo, estilo Inca Asociado. Desde las investigaciones etnohistóricas realizadas principalmente por Rostworowski, en la década de 1970, en la actualidad el término Ichma viene siendo asumido para la designación del hábeas cerámico local, previo a la ocupación Inca de los valles bajos del Rimac, Lurín y Chilca. 